prehypertensive patients, and increased by $12.0 \mathrm{~mm}^{3}$ in hypertensive patients $(P<0.05$ for all comparisons).

These results indicate that there is a continuous relationship between BP and progression of coronary atherosclerosis. As patients with normal BP showed the slowest progression rate, and even regression of atherosclerosis, the authors conclude that, for patients with coronary disease, BP goals should be substantially lower than the $<140 / 90 \mathrm{mmHg}$ level recommended in current guidelines.

Original article Sipahi I et al. (2006) Effects of normal, pre-hypertensive, and hypertensive blood pressure levels on progression of coronary atherosclerosis. J Am Coll Cardiol 48: 833-838

\section{CARE-HF extension demonstrates a sustained benefit of CRT}

The Cardiac Resynchronization in Heart Failure (CARE-HF) trial investigated the benefits of cardiac resynchronization therapy (CRT) in patients with persistent moderate or severe symptoms of heart failure. This open-label randomized trial assigned patients to either medical therapy alone $(n=404)$ or medical therapy with implantation of a CRT device $(n=409)$. After a mean follow-up of 29.4 months, CRT produced benefits over medical therapy alone in terms of reductions in morbidity and mortality, as well as improving cardiac function, symptoms and quality of life.

The initial expense of CRT is higher than that of medical therapy, and implantation is associated with some morbidity; however, CRT has previously been shown to be cost-effective. The CARE-HF trial was extended for a further 8 months after completion of the main study, to determine whether the benefits of CRT remain over the long term. Over a mean follow-up of 37.4 months, the mortality in the medical therapy group was $38.1 \%$, compared with $24.7 \%$ in the CRT group. The authors calculate that CRT would result in 1 additional patient being alive at 2 years for every 13 patients in whom device implantation is attempted, rising to 1 in every 9 at 3 years. The decrease in mortality in the CRT group resulted from a reduction in sudden death and in death caused by worsening heart failure. Results from the CARE-HF trial extension demonstrate that the benefits of CRT persist and might even increase over time.
Original article Cleland JGF et al. (2006) Longer-term effects of cardiac resynchronization therapy on mortality in heart failure [the Cardiac Resynchronization-Heart Failure (CARE-HF) trial extension phase]. Eur Heart J 27: 1928-1932

\section{Elevated serum retinol-binding protein 4 is a marker of insulin resistance}

Elevated serum levels of retinol-binding protein 4 (RBP4), a retinol transporter secreted by adipocytes and liver, are associated with insulin resistance and diabetes. Graham et al., therefore, sought to determine the relationship between RBP4 and insulin resistance, and whether intervention to increase insulin sensitivity influenced RBP4 levels.

Serum RBP4 levels were found to correlate positively with insulin resistance in obese individuals, those with impaired glucose tolerance, and those with type 2 diabetes. The same correlation was found in nonobese, nondiabetic individuals who had a family history of diabetes. RBP4 levels correlated with $\mathrm{BMI}$, but the relationship between RBP4 and insulin resistance was independent of obesity; nonobese insulin-resistant individuals also had elevated levels of RBP4. Four weeks of exercise training in individuals with type 2 diabetes or impaired glucose tolerance produced an increase in insulin sensitivity in two-thirds of participants. Notably, an increase in insulin sensitivity was accompanied by a decrease in RBP4 levels. In participants who experienced marginal or no improvement in insulin resistance, however, RBP4 levels increased or remained unchanged. Elevated RBP4 levels were associated with increased waist:hip ratio, increased serum triglyceride levels, increased systolic blood pressure and decreased HDL cholesterol levels, all of which are elements of the metabolic syndrome.

The authors state that measurement of serum RBP4 could provide a means of assessing the risk of insulin resistance, type 2 diabetes and cardiovascular disease. As elevated RBP4 levels have been shown to cause insulin resistance in mice, the authors speculate that RBP4-reducing therapy might decrease insulin resistance in humans.

\footnotetext{
Original article Graham TE et al. (2006) Retinol-binding protein 4 and insulin resistance in lean, obese, and diabetic subjects. N Engl J Med 354: 2552-2563
} 